TRANSACTIONS OF THE

AMERICAN MATHEMATICAL SOCIETY

Volume 355, Number 9, Pages 3475-3484

S 0002-9947(03)03323-3

Article electronically published on May 29, 2003

\title{
REMARKS ABOUT UNIFORM BOUNDEDNESS OF RATIONAL POINTS OVER FUNCTION FIELDS
}

\author{
LUCIA CAPORASO
}

\begin{abstract}
We prove certain uniform versions of the Mordell Conjecture and of the Shafarevich Conjecture for curves over function fields and their rational points.
\end{abstract}

\section{INTRODUCTION AND PRELIMINARIES}

A curve $X$ of genus at least 2 defined over a function field $L$ has only finitely many $L$ rational points, unless it is isotrivial. Similarly, a curve of genus at least 2 defined over a number field $F$ has a finite set of $F$-rational points. These well-known facts are celebrated theorems of Y. Manin and G. Faltings, originally conjectured by L. J. Mordell and S. Lang.

We study here questions of uniformity for the cardinality of such sets of rational points, in the function field case. For number fields, there are a number of open conjectures, such as the following (Uniform Mordell Conjecture for number fields): Fix $g \geq 2$ and a number field $F$; there exists a number $B_{g}(F)$ such that any curve of genus $g$ defined over $F$ has at most $B_{g}(F)$ rational points over $F$. Interest in such problems was revived after it was proved in $\mathrm{CHM}$ that the conjecture above is a consequence of a famous, open, conjecture (usually attributed to S. Lang and E. Bombieri) on the non-density of rational points in varieties of general type (see also $\mathrm{Ab}, \mathrm{AV}]$ and $\mathrm{Pa}]$ ).

In this paper we investigate similar issues for curves over function fields. Some partial results were obtained in [Mi] and in [C] were the existence of uniform bounds for the sets of rational points is established. Such bounds depend on suitable numerical invariants of the function field, on the genus $g$ of the curves and on the degree of the locus of bad reduction (that is, the locus of singular fibers).

We shall also study here the strictly related "uniform Shafarevich problem"; a famous theorem of A. N. Parshin and S. Ju. Arakelov ( $[\mathrm{Ar}]$ and $[\mathrm{P}]$ ) states that if $B$ is a smooth complex curve and $S \subset B$ a finite subset, then there exists only a finite number of non-isotrivial families of smooth curves of fixed genus $g \geq 2$ over $B-S$. Parshin first proved it under the assumption that $S=\emptyset$; Arakelov generalized it a few years later. In [P] Parshin shows also that the above theorem implies finiteness of rational points for non-isotrivial curves of genus at least 2, providing the above mentioned link between the Shafarevich problem and the Mordell problem. Recall that his argument, known as the "Parshin trick", is valid for both number fields and function fields.

Received by the editors January 10, 2001 and, in revised form, September 24, 2001.

2000 Mathematics Subject Classification. Primary 14H05, 14H10.

(C)2003 American Mathematical Society 
A first uniform version of the theorem of Parshin and Arakelov above is obtained in $[\mathrm{C}]$. We here generalize it by a stronger uniform result valid for families of curves over bases of any dimension. This is done in Section 2, where we obtain bounds (for the sets of curves with fixed degeneracy locus as well as for the sets of rational points) that only depend on the degree of a polarization on the base variety, and on the degree of the locus of bad reduction. A stronger result can be obtained for curves having good reduction in codimension 1 (Theorem 3). In Section 3 we will consider families with maximal variation of moduli, using the geometry of the moduli space of curves to approach our problems.

We work over $\mathbb{C}$; by $V$ we shall denote a smooth, irreducible, projective variety over $\mathbb{C}$, whose field of rational functions will be $L:=\mathbb{C}(V)$. Special interest will be given to varieties of dimension 1 , for which we shall use the following notation: $B$ is a smooth irreducible curve and $K$ its field of rational functions. We fix integers $q \geq 0, g \geq 2$ and $s \geq 0$ throughout. The genus of $B$ will be denoted by $q$.

We shall consider smooth curves of genus $g$ over the function field $L$ (or $K$ ), which can also be viewed as families of curves over $V$, such that there is a nonempty open subset of $V$ over which the fibers are all smooth. We shall always assume that such a family (or curve) is not isotrivial, i.e., the smooth fibers are not all isomorphic.

To be more precise, we introduce the following sets: let $B$ be a fixed curve and let $S \subset B$ be a finite set of points.

Definition. $F_{g}(B, S)$ shall denote the set of equivalence classes of non-isotrivial families $f: X \longrightarrow B$ such that $X$ is a smooth relatively minimal surface and the fiber $X_{b}$ over every $b \notin S$ is a smooth curve of genus $g$. Two such families $f_{i}: X_{i} \longrightarrow B$ for $i=1,2$ are equivalent if there is a commutative diagram

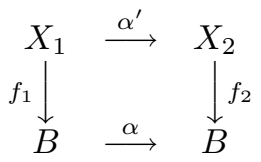

where the two horizontal arrows are birational maps.

Using a different terminology, $F_{g}(B, S)$ is the set of $K$-isomorphism classes of non-isotrivial curves of genus $g$ over $K$, having good reduction outside of $S$. The theorem of Parshin and Arakelov says that $F_{g}(B, S)$ is finite. Theorem 3.1 of [C] states that there exists a number $P(g, q, s)$ such that $\left|F_{g}(B, S)\right| \leq P(g, q, s)$ for every curve $B$ of genus $q$ and for every subset $S$ having at most $s$ points. We show here (in the end of Section 2) that this result is sharp in the sense that such a bound must depend on $s$.

We are interested in function fields of higher transcendence degree. We can generalize the definition of $F_{g}(B, S)$ as follows. Let $T \subset V$ be a closed subscheme.

Definition. $F_{g}(V, T)$ shall be the set of equivalence classes of non-isotrivial families of smooth curves of genus $g$ over $V-T$ (the equivalence relation is the same as above, with $B$ replaced by $V-T$ ).

By the existence and unicity of minimal models for smooth surfaces, this definition coincides with the previous one if $\operatorname{dim} V=1$. It follows from the results in [C] (3.4) that $F_{g}(V, T)$ is finite. Our best result on $F_{g}(V, T)$ is Theorem 1.

If $X$ is a curve defined over a field $L$, we shall denote by $X(L)$ the set of its $L$-rational points. If $X$ has genus at least 2 and it is not isotrivial, the theorem of Manin says that $X(L)$ is finite. Consider now the Uniformity Conjecture for 
rational points over function fields, which can be stated as its arithmetic analogue: Let $L$ be a function field over $\mathbb{C}$ and let $g \geq 2$ be an integer. There exists a number $N_{g}(L)$ such that for every non-isotrivial curve $X$ of genus $g$ defined over $L$ we have $|X(L)| \leq N_{g}(L)$. For results relating it to the Lang Conjectures about the distributions of rational points on varieties of general type, see the work of D. Abramovich and J. F. Voloch AV.

Such a conjecture remains open; our results in that direction are Theorems 2 and 3 and Proposition 4.

A final piece of notation. $M_{g}$ denotes the moduli variety of smooth curves of genus $g$ and $\bar{M}_{g}$ its compactification via Deligne-Mumford stable curves. They are both integral, normal varieties of dimension $3 g-3$. A universal curve exists only on a proper open subset of $M_{g}$ (and of $\bar{M}_{g}$ ). In particular, a morphism $\phi: Z \longrightarrow M_{g}$ does not necessarily come from a family of curves over $Z$. If this is the case, that is, if there exists a family of smooth curves $X \longrightarrow Z$ such that for every $z \in Z, \phi(z)$ is the isomorphism class of the fiber of $X$ over $z$, we shall say that $\phi$ is a moduli map.

\section{UNIFORMITY RESULTS FOR FUNCTION FIELDS OF HIGH TRANSCENDENCE DEGREE}

We start by a uniform generalization of the theorem of Parshin and Arakelov. The result below is a strenghtening of 3.4 and 3.5 in [C]; in fact, the bound $H$ here is independent of the dimension of $V$ and of $r$. Such an improvement is obtained by a small technical modification of the methods in [C].

Notice that the statement below remains true if $V$ is replaced by an integral, possibly singular, projective variety. The proof is essentially the same.

Theorem 1. Let $g \geq 2, d \geq 1, s \geq 0$ be fixed integers. There exists a number $H(g, d, s)$ such that for any smooth, irreducible variety $V \subset \mathbb{P}^{r}$ of degree $d$, for any closed subscheme $T \subset V$ of degree $s$, we have $\left|F_{g}(V, T)\right| \leq H(g, d, s)$. Moreover, if $T$ has codimension at least 2 in $V$, then the bound $H$ does not depend on $s$.

Proof. Step 1: Slicing V into curves of bounded genus. Considering one-dimensional hyperplane sections of $V$, we see that $V$ can be covered by smooth curves of degree $d$ passing through any of its points; it is a well-known fact that the genus of a curve of degree $d$ in projective space is at most equal to $\left(\begin{array}{c}d-1 \\ 2\end{array}\right)$ : just project the curve birationally onto a curve of degree $d$ in $\mathbb{P}^{2}$. Let

$$
q=q(d)=\left(\begin{array}{c}
d-1 \\
2
\end{array}\right)
$$

so that $V$ is covered by curves of geometric genus at most $q$.

Step 2: Uniform boundedness of moduli maps. By Theorem 3.1 in [C], for any fixed $g, q^{\prime}$, and $s^{\prime}$ there exists a number $P\left(g, q^{\prime}, s^{\prime}\right)$ such that for any smooth curve $B$ of genus $q^{\prime}$, for any subset $S \subset B$ of at most $s^{\prime}$ points, we have that $\left|F_{g}(B, S)\right| \leq$ $P\left(g, q^{\prime}, s^{\prime}\right)$.

Define

$$
H^{\prime}=\max _{q^{\prime} \leq q, s^{\prime} \leq s} P\left(g, q^{\prime}, s^{\prime}\right)
$$

so that $H^{\prime}$ only depends on $g, d, s$; let $U=V-T$. 
We claim that $U$ has at most $H^{\prime}$ moduli maps to $M_{g}$; that is, we claim that there exist at most $H^{\prime}$ non-constant, (regular) morphisms $\phi: U \longrightarrow M_{g}$ such that there exists a (not necessarily unique, see below) family of smooth curves over $U$ whose moduli map is $\phi$. By contradiction, let $n>H^{\prime}$ and let us assume that there exist $\phi_{1}, \ldots, \phi_{n}$ distinct such moduli maps $\phi_{i}: U \longrightarrow M_{g}$. Let $X_{i} \longrightarrow U$ be a non-isotrivial family of smooth curves corresponding to $\phi_{i}$ (since $\phi_{i}$ is a moduli map, such a family exists, but it is not necessarily unique). Let $U^{\prime} \subset U$ be the nonempty open subset where $\phi_{i}(u) \neq \phi_{j}(u)$ for every $u \in U^{\prime}$ and for every pair of distinct $i, j$. Let $p \in U^{\prime}$ and let $F_{i}=\phi_{i}^{-1} \phi_{i}(p)$; since $\phi_{i}$ is not constant, its fiber $F_{i}$ through $p$ is a proper closed subset of $U^{\prime}$. Therefore, there exists a curve $B \subset V$ of genus at $q^{\prime} \leq q$ such that $p \in B$ and such that $B \not \subset F_{i}$ for every $i=1, \ldots, n$; thus the restriction of $X_{i}$ to $B$ is not isotrivial for every $i$. Let $S=(B \cap T)_{\text {red }}$. Let $Y_{i} \longrightarrow B$ be the smooth relatively minimal completion over $B$ of the restriction of $X_{i}$ to $B$. By construction, $Y_{1}, \ldots, Y_{n}$ are different elements of $F_{g}(B, S)$, which is a contradiction, since $F_{g}(B, S)$ has at most $P\left(g, q^{\prime}, s^{\prime}\right) \leq H^{\prime}<n$ elements. This proves the claim. Notice that if $T$ has codimension at least 2 in $V$ we can always choose our $B$ so that it does not intersect $T$ at all, and hence $S$ can be taken to be the empty set and $H^{\prime}$ does not depend on $s$.

Conclusion. Given a moduli map $\phi: U \longrightarrow M_{g}$ the set of families that have $\phi$ as moduli map is uniformly bounded; in fact, it is bounded above by a function of $g$ only (see [C], Lemma 3.3); hence we are done.

A similar argument yields the following uniformity statement for rational points, stronger than 4.3 and 4.4 in $[\mathrm{C}]$ :

Theorem 2. Let $g \geq 2, d \geq 1, s \geq 0$ be fixed integers. There exists a number $N(g, d, s)$ such that for any smooth, irreducible variety $V \subset \mathbb{P}^{r}$ of degree $d$, for any closed subscheme $T \subset V$ of degree $s$ and for any non-isotrivial curve $X$ of genus $g$ defined over $L=\mathbb{C}(V)$ and having good reduction outside of $T$, we have $|X(L)| \leq N(g, d, s)$. Moreover, if $T$ has codimension at least 2 in $V$, then the bound $N$ does not depend on $s$.

Proof. Step 1: Repeat word for word Step 1 in the proof of the previous theorem.

Step 2: Theorem 4.2 in $\left[\mathrm{C}\right.$ says that if $g, q^{\prime}$, and $s^{\prime}$ are fixed nonnegative integers, there exists a number $M\left(g, q^{\prime}, s^{\prime}\right)$ such that for any curve $B$ of genus $q^{\prime}$, for any subset $S$ of at most $s^{\prime}$ points in $B$, and for any curve $X_{B} \in F_{g}(B, S)$ we have that

$$
\left|X_{B}(\mathbb{C}(B))\right| \leq M\left(g, q^{\prime}, s^{\prime}\right) .
$$

Arguing as in the proof of 4.4 of [C] one gets that defining

$$
N(g, d, s):=\max _{q^{\prime} \leq q, s^{\prime} \leq s} M\left(g, q^{\prime}, s^{\prime}\right)
$$

will suffice for our statement.

To conclude, we show that for curves having good reduction in codimension 1 , stronger finiteness results hold. Let $L$ be a function field over $\mathbb{C}$ and let $V$ be a smooth, projective, complex variety of positive dimension such that $L=\mathbb{C}(V)$.

Definition. Let $C_{g}^{2}(L)$ be the set of $L$-isomorphism classes of non-isotrivial curves of genus $g$ over $L$ having good reduction in codimension 1 .

In other words, $C_{g}^{2}(L)$ is the set of equivalence classes of non-isotrivial families $X \longrightarrow V$ of curves of genus $g$ over $V$ such that there exists a closed subscheme 
$T \subset V$ of codimension at least 2 with the property that $X_{v}$ is smooth for every $v \notin T$.

\section{Theorem 3.}

a) $C_{g}^{2}(L)$ is finite.

b) There exists a number $N_{g}^{2}(L)$ such that for every curve $X \in C_{g}^{2}(L)$ we have $|X(L)| \leq N_{g}^{2}(L)$.

Proof. We shall use moduli maps. Denote by $M_{g}^{2}(L)$ the set of equivalence classes of non-constant rational maps $\phi: V \longrightarrow M_{g}$ such that there exists an open subset $U^{\phi} \subset V$ with the following properties:

1. The complement of $U^{\phi}$ has codimension at least 2 in $V$.

2. $\phi$ is regular on $U^{\phi}$.

3. There exists a (non-isotrivial) family of smooth curves of genus $g$ over $U^{\phi}$ such that $\phi$ is its moduli map.

4. Two such maps $\phi$ and $\psi$ are equivalent iff they coincide on some (nonempty) open subset of $V$.

There is a natural surjective map of sets:

$$
\mu: C_{g}^{2}(L) \longrightarrow M_{g}^{2}(L)
$$

sending a curve over $L$ to its moduli map (it is easy to see that $\mu$ is well defined). Now, $\mu$ has finite fibers (Lemma 3.3 in [C]) and is surjective by definition. Thus $C_{g}^{2}(L)$ is finite if and only if $M_{g}^{2}(L)$ is finite.

Part b) is an immediate consequence of part a), by the theorem of Manin. We will prove our result by showing that $M_{g}^{2}(L)$ is finite by induction on $\operatorname{dim} V$. If $\operatorname{dim} V=1$, then the finiteness of $C_{g}^{2}(L)$ and of $M_{g}^{2}(L)$ is the theorem of Parshin (the locus of bad reduction being empty in such a case). Then let $\operatorname{dim} V \geq 2$ and suppose that $M_{g}^{2}(L)$ is infinite. Notice that $M_{g}^{2}(L)$ is dominated by a union of finite sets as follows: if $T$ is a closed subset of $V$, denote by $M_{g}(V, T)$ the set of equivalence classes of moduli maps to $M_{g}$ that are regular on $V-T$; then $M_{g}(V, T)$ is finite, by Theorem 1 and Lemma 3.3 in [C]. We have a natural, surjective map

$$
\bigcup_{\operatorname{codim}_{V} T \geq 2} M_{g}(V, T) \longrightarrow M_{g}^{2}(L)
$$

hence, if $M_{g}^{2}(L)$ is infinite, so is the union on the left-hand side. Then there exists a countable collection $\left\{T_{n}, n \in \mathbb{Z}\right\}$, with $T_{n}$ a closed subset of $V$ of codimension at least 2 , such that the set

$$
M:=\bigcup_{n \in \mathbb{Z}} M_{g}\left(V, T_{n}\right)
$$

is infinite. Now, $M$ itself being a countable set, we shall put an ordering on it:

$$
M=\left\{\phi^{i}, i \in \mathbb{N}\right\} .
$$

For every pair of distinct $i, j$, denote by $U^{i, j}$ the nonempty open subset of $V$ such that $U^{i, j} \subset U^{\phi^{i}} \cap U^{\phi^{j}}$ and $\phi^{i}(u) \neq \phi^{j}(u)$ for every $u \in U^{i, j}$. The $U^{i, j} \mathrm{~S}$ form a countable collection of nonempty open subsets of $V$, whose intersection $I$ is dense in $V$. Let $p \in I$ and, for every $i \in \mathbb{N}$, let $F_{i}=\left(\phi^{i}\right)^{-1} \phi^{i}(p)$ be the fiber of $\phi^{i}$ through $p$. Since $\phi^{i}$ is non-constant (by assumption), $F_{i}$ is a proper closed subset of $V$. Thus, the complement of $\bigcup_{i \in \mathbb{N}} F_{i}$ intersects $I$ in a subset $J$, with $J$ dense in $V$. Fix a (non-degenerate) projective model of $V$ in some projective space. Then there 
exists a hyperplane $H$ such that $p \in H$, such that $H \cap J \neq \emptyset$ and such that $H$ does not contain any $T_{n}$. Letting $W=H \cap V$, we can furthermore choose $H$ so that $W$ is smooth. By construction we have

(a) $\operatorname{dim} W=\operatorname{dim} V-1$;

(b) $\operatorname{dim} T_{n} \cap W=\operatorname{dim} T_{n}-1 \leq \operatorname{dim} W-2$ (since $H$ does not contain any $T_{n}$ );

(c) $\forall \phi^{i} \in M$, the restriction $\phi_{\mid W}^{i}$ is not constant (since $p \in W$ and $W \cap J \neq \emptyset$ );

(d) $\forall i \neq j$ we have $\phi_{\mid W}^{i} \neq \phi_{\mid W}^{j}$ (since $W \cap I \neq \emptyset$ );

hence $\phi_{\mid W}^{i} \in M_{g}^{2}(\mathbb{C}(W))$ and the restriction to $W$ gives an inclusion (by (d) above) $M \hookrightarrow M_{g}^{2}(\mathbb{C}(W))$. Thus $M_{g}^{2}(\mathbb{C}(W))$ is infinite. This is a contradiction with the inductive assumption.

See $[\mathrm{Md}]$ for an analogue over $\mathbb{Q}$. Part a) of this result should be compared with the examples of A. Beauville (in [B], section 5) or with the example below. They show that the assumption that the curves have good reduction in codimension 1 is crucial; that is, a) is false without that assumption. On a different vein, compare also with Proposition 4. The example that we are going to describe shows that there is no hope of getting a substantially stronger uniform version of the Shafarevich Conjecture for function fields; in other words, any uniform bound on $\left|F_{g}(B, S)\right|$ must depend on the degree of $S$.

What happens to the cardinality of $F_{g}(B, S)$ when $s$ grows while $g$ and $q$ (or even $B$ ) stay fixed? The way we defined $F_{g}(B, S)$, it is an exercise to show that its cardinality is not bounded; but this is just because the families parametrized by $F_{g}(B, S)$ are not required to have a singular fibers over $S$. The interesting question is about the asymptotics of the cardinality of that subset of $F_{g}(B, S)$ parametrizing families of curves that have a singular fiber over every point of $S$. We will make this precise now, describing an example suggested by J. de Jong, showing that the set of fibrations with fixed degeneracy locus is not bounded, as the cardinality of the degeneracy locus grows.

Fix $g \geq 2$ and $B=\mathbb{P}^{1}$. Given a subset $S \subset \mathbb{P}^{1}$ denote by $F(S) \subset F_{g}\left(\mathbb{P}^{1}, S\right)$ the set of all genus $g$ non-isotrivial fibrations $X \longrightarrow \mathbb{P}^{1}$ such that the fiber $X_{b}$ is smooth if and only if $b \notin S$.

Let $S=\left\{a_{1}, \ldots . a_{s}\right\}$ be a set of generic points in $\mathbb{P}^{1}$, and let $I \cup J=\{1,2, \ldots ., s\}$ be a partition of $\{1,2, \ldots, s\}$ in two disjoint subsets such that $|I|=5$. Define a non-isotrivial fibration $X_{I}$ of curves of genus 2 over $\mathbb{P}^{1}$ by the affine equation

$$
y^{2}=(x-t) \Pi_{i \in I}\left(x-a_{i}\right) \Pi_{j \in J}\left(t-a_{j}\right)
$$

with $t$ an affine coordinate in $\mathbb{P}^{1}$. For $t \notin S$ (and $t \neq \infty$ ) we get a smooth curve of genus 2. For $t=a_{i}$ with $i \in I$, we get a nodal curve and for $t=a_{j}, j \in J$, we get a singular, non-reduced curve. Thus $X_{I} \in F(S \cup \infty)$ and, by varying the partition $I \cup J$, we get a total of $\left(\begin{array}{c}s \\ 5\end{array}\right)$ different such fibrations. Hence the cardinality of $F(S)$ goes to infinity, as $|S|$ grows. 
One final word about this example.

First, we make two comments: the given family has fibers of genus 2, but of course one can construct the same example for any genus (just replace the integer 5 by a higher odd number), obtaining families of hyperelliptic curves.

The second comment is about those singular fibers over $a_{j}$ with $j \in J$ that are not stable curves; their semistable reduction is actually a smooth curve. The remaining 5 fibers over $a_{i}$ are instead nodal. In other words, the moduli map $\phi_{I}$ associated to the family $X_{I} \longrightarrow \mathbb{P}^{1}$,

$$
\phi_{I}: \mathbb{P}^{1} \longrightarrow \overline{M_{2}}
$$

(such that $\phi_{I}(t)$ is the isomorphism class of the fiber of $X_{I}$ over $t$ ) intersects the boundary $\Delta_{2}$ in exactly 5 points, regardless of the cardinality of $S$.

We ask:

(a) Can one find similar examples whose fibers do not belong to any proper closed subset of $M_{g}$ ?

(b) Is the same "unboundedness" result true for families of stable curves? In other words, does there exist a similar example all of whose singular fibers are nodal?

\section{UNIFORMITY FOR "TRULY VARYING" CURVES}

This section contains results that are independent of the degeneracy locus. Given a family $X \longrightarrow V$ of generically smooth curves of genus $g$ over $V$, we get a natural rational map $\phi: V \longrightarrow M_{g}$ (regular on a nonempty open subset of $V$ ). The dimension of the image of $\phi$ is called the variation of moduli of the family; we shall say that the family has maximal variation of moduli if

$$
\operatorname{dim} \operatorname{Im} \phi=\min \{\operatorname{dim} V, 3 g-3\} .
$$

We shall say that a curve over $L=\mathbb{C}(V)$ has maximal variation of moduli if a corresponding family of curves over $V$ does.

Thus the condition of having maximal variation of moduli can be interpreted as saying that the family (or the curve) is truly varying and can be viewed as a generalization of the non-isotriviality condition. Obviously, if the base field has transcendence degree 1 , a curve is non-isotrivial if and only if it has maximal variation of moduli.

Definition. Let $L$ be a function field. We define $C_{g}(L)$ to be the set of $L$-isomorphism classes of curves of genus $g$ defined over $L$ and having maximal variation of moduli.

Proposition 4. Let $g \geq 24$ and let $L$ be a function field of transcendence degree $3 g-3$. Then

a) $C_{g}(L)$ is finite.

b) There exists a number $N(L, g)$ such that for every curve $X$ of genus $g$ defined over $L$ and having maximal variation of moduli, we have $|X(L)| \leq$ $N(L, g)$.

c) There exists a function $P_{g}(n, m)$ such that for every $V$ of general type, we have $\left|C_{g}(L)\right| \leq P_{g}\left(\operatorname{dim} V, K_{V}^{\operatorname{dim} V}\right)$.

Proof. The assumption $g \geq 24$ implies that $\bar{M}_{g}$ is of general type (for this famous result of J. Harris and D. Mumford we refer to $[\mathrm{HMu}]$ and to $6 \mathrm{~F}$ in $[\mathrm{HM}]$ ). 
Denote by $R\left(V, \bar{M}_{g}\right)$ the set of dominant, rational maps from $V$ to $\bar{M}_{g}$. A theorem of Kobayashi and Ochiai $\left[\mathrm{KO}\right.$ implies that, $\bar{M}_{g}$ being of general type, $R\left(V, \bar{M}_{g}\right)$ is finite. Notice now that there is a natural bijection between $C_{g}(L)$ and $R\left(V, \bar{M}_{g}\right)$ : to a truly varying curve $X$ of genus $g$ over $L$ we can associate its moduli map $\phi_{X} \in R\left(V, \bar{M}_{g}\right)$. The fact that such a correspondence is bijective follows from the existence of the universal curve over an open subset of $\bar{M}_{g}$. Thus $C_{g}(L)$ is finite.

By the theorem of Manin, any curve in $C_{g}(L)$ has a finite set of $L$-rational points. Thus part b) follows immediately from a).

Part c) is proved like part a); we can in this case apply a strengthening of the theorem of Kobayashi and Ochiai provided by T. Bandman and D. Markushevich. From $[\overline{\mathrm{BM}}]$ we obtain that, $V$ and $\bar{M}_{g}$ being of general type and $\bar{M}_{g}$ having canonical singularities (Theorem 1 in $[\mathrm{HMu}]$ ), there exists a function of $g$, of $\operatorname{dim} V$ and of $K_{V}^{\operatorname{dim} V}$ bounding the cardinality of $R\left(V, \bar{M}_{g}\right)$ and hence that of $C_{g}(L)$.

Let $u: \mathcal{C}_{g} \longrightarrow M_{g}^{o}$ be the universal curve over the moduli space of automorphism free smooth curves of genus $g$, so that the fiber of $u$ over the point corresponding to the curve $X$ is $X$ itself.

It is a well-known fact (see [HM], 2D) that $u$ has no rational sections; thus, $\mathcal{C}_{g}$ has no rational point over the function field of $M_{g}$. In fact, much more is known: the Picard group of $\mathcal{C}_{g}$ is generated over the Picard group of $M_{g}$ by the relative dualizing sheaf $\omega_{u}$; therefore, a multisection of $u$ must have degree over $M_{g}^{o}$ equal to a multiple of $2 g-2$.

We apply this to obtain that if $V$ is a variety of dimension $3 g-3$ and $X$ is a curve of genus $g$ over $L$ having maximal variation of moduli, then a necessary condition for $X$ to have a rational point over $L$ is that its moduli map have degree equal to a multiple of $2 g-2$. This follows easily by looking at the commutative diagram

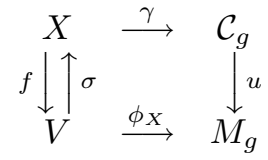

where the horizontal arrows are rational maps and $\sigma$ is the rational section corresponding to a rational point of $X$ over $L$. Let $\tau=\gamma \circ \sigma: V \longrightarrow \mathcal{C}_{g}$ and let $\rho: \operatorname{Im} \tau \longrightarrow M_{g}$; by what we said, $\operatorname{deg} \rho=n(2 g-2)$ for some integer $n$. We finally obtain

$$
\operatorname{deg} \phi_{X}=\operatorname{deg} \tau \cdot \operatorname{deg} \rho=m(2 g-2),
$$

where by $\operatorname{deg} \phi_{X}$ we mean the degree of the restriction of $\phi_{X}$ to the nonempty open subset of $V$ and where $\phi_{X}$ is a regular and finite map. Let us call such a number $\operatorname{deg} \phi_{X}$ the modular degree of a family $X \longrightarrow V$; this definition is general, provided that $X \longrightarrow V$ has maximal variation of moduli and that $\operatorname{dim} V \leq 3 g-3$. We just proved the following

Lemma 5. Let $V$ be a variety of dimension $3 g-3$ with function field $L$ and let $X$ be a smooth curve of genus $g$ over $L$ having maximal variation of moduli. Then either $X(L)=\emptyset$ or the modular degree of $X$ is a multiple of $2 g-2$. 
The following well-known conjecture is open:

Geometric Lang Conjecture. Let $W$ be a variety of general type defined over $\mathbb{C}$. Then there exists a proper closed subvariety $Z_{W}$ of $W$ containing all positivedimensional subvarieties of $W$ that are not of general type.

In particular, according to such a conjecture, all curves in $W$ having genus at most 1 are contained in $Z_{W}$.

Consider now $\bar{M}_{g}$, and let $Z_{g} \subset \bar{M}_{g}$ be defined as the closure of the union of all integral curves in $\bar{M}_{g}$ having geometric genus at most equal to 1 . Since $\bar{M}_{g}$ is of general type if $g \geq 24$, the above conjecture would imply that $Z_{g}$ is a proper, closed subset of $\bar{M}_{g}$ for all $g \geq 24$.

As a consequence, we get the following.

Lemma 6. Let $g \geq 24$ and let $B$ be a curve of genus $q$. The Geometric Lang Conjecture implies that if $X \longrightarrow B$ is a non-isotrivial family of curves of genus $g$, passing through the general point of $M_{g}$, then the modular degree of $X$ is at most $q-1$.

Proof. As we mentioned above, the union of all curves in $\bar{M}_{g}$ of genus at most 1 is contained in a proper closed subset $Z_{g}$ of $\bar{M}_{g}$. The condition that the given family of curves goes through the general point of $\bar{M}_{g}$, combined with the Geometric Lang Conjecture, implies that $\operatorname{Im} \phi_{X} \not \subset Z_{g}$. Thus the geometric genus of $\operatorname{Im} \phi_{X}$ is at least 2. By the Riemann-Hurwitz formula, the degree $d$ of a dominant map of a curve $B$ of genus $q$ onto a curve $C$ of geometric genus $p \geq 2$ is at most equal to $q-1$. In fact, the formula gives

$$
d=\frac{2 q-2-r}{2 p-2} \leq \frac{q-1}{p-1} \leq q-1
$$

since $r \geq 0$ (being the degree of the ramification divisor) and $p \geq 2$ by assumption.

\section{ACKNOWLEDGMENTS}

I am grateful to Olivier Debarre and to Johan de Jong for useful conversations and to Felipe Voloch for indicating relevant references. Special thanks to Dan Abramovich for pointing out a serious mistake in a previous version of this paper.

\section{REFERENCES}

[Ab] D. Abramovich: Uniformity of stably integral points on elliptic curves, Inventiones Math. 127, 307-317 (1997). MR 98d:14033

[AV] D. Abramovich and J. F. Voloch: Lang's conjectures, fibered powers, and uniformity, New York J. Math. 2, 20-34 (1996) MR 97e:14031

[Ar] S. Ju. Arakelov: Families of algebraic curves with fixed degeneracies, Izv. Akad. Nauk. SSSR Ser. Mat. 35, 1269-1293 (1971). MR 48:298

[BD] T. Bandman and G. Dethloff: Estimates of the number of rational mappings from a fixed variety to varieties of general type, Ann. Inst. Fourier (Grenoble) 47 (1997), no. 3, 801-824. MR 98h:14016

[BM] T. Bandman and D. Markushevich: On the number of rational maps between varieties of general type, J. Math. Sci. Univ. Tokyo 1 (1994), no. 2, 423-433. MR 96c:14012

[B] A. Beauville: Exposé No. 6 in Séminaire sur les pinceaux des courbes de genre au moins deux, Astérisque 86 (1981). MR 83c:14020

[C] L. Caporaso: On certain uniformity properties of curves over function fields, Preprint, AG/9906156, Compositio Mathematica 130 (2002), 1-19. MR 2003a:14038 
[CHM] L. Caporaso, J. Harris, and B. Mazur: Uniformity of rational points, J. Amer. Math. Soc. 10 (1997), 1-35. MR 97d:14033

[DF] M. De Franchis: Un teorema sulle involuzioni irrazionali, Rend. Circ. Mat. Palermo 36 (1913), 368.

[HM] J. Harris and I. Morrison: Moduli of curves, Graduate Texts in Mathematics 187, SpringerVerlag, New York, 1998. MR 99g:14031

$[\mathrm{HMu}] \mathrm{J}$. Harris and D. Mumford: On the Kodaira dimension of the moduli space of curves, Inventiones Math. 67 (1982), 23-88. MR 83i:14018

[KO] S. Kobayashi and T. Ochiai: Meromorphic mappings onto compact complex spaces of general type, Inventiones Math. 31 (1975), 7-16. MR 53:5948

[Md] M. Martin-Deschamps: Conjecture de Shafarevich pour les corps de fonctions sur $Q$, Astérisque No. 127, Appendice à l'exposé IX (1985), 256-259.

[Ma] Y. Manin: Rational points of algebraic curves over function fields, Izv. Akad. Nauk. SSSR Ser. Mat. 27 (1963), 1395-1440. MR 28:1199

[Mi] Y. Miyaoka: Themes and variations on inequalities of Chern classes, Sugaku 41 (1989), 193-207. MR 91j:14003

[Pa] P. Pacelli: Uniform boundedness for rational points, Duke Math. J. 88 (1997), 77-102. MR 98b:14020

[P] A. N. Parshin: Algebraic curves over function fields, Izv. Akad. Nauk. SSSR Ser. Mat. 32 (1968), 1191-1219. MR 41:1740

[S] L. Szpiro: Exposé No. 3 in Séminaire sur les pinceaux des courbes de genre au moins deux, Astérisque 86 (1981). MR 83c:14020

Department of Mathematics, Università degli Studi Roma III, Largo San Leonardo Murialdo 1, 00146 Rome, Italy

E-mail address: caporaso@mat.uniroma3.it 